\title{
Pratiques Alimentaires Des Mères Et Malnutrition Infantile Dans Le District De Santé De Pitoa: Etude Cas-Témoin
}

\author{
Benjamin Azike Chukuwchindun'1, André Pascal Goura ${ }^{1}$, Landry Bita'a Beyala ${ }^{1}$, \\ Anthony Njimbia Chebe ${ }^{1}$, André Izacar Gaël Bita ${ }^{4}$, Joliette Azakoh Nguefack ${ }^{1}$, Jérôme \\ Ateudjieu ${ }^{1,2,3}$ \\ ${ }^{1}$ ONG Meilleur Accès Aux Soins de SANTE (M.A. SANTE), Cameroun \\ ${ }^{2}$ Université de Dschang, Filière des Sciences Biomédicales, Chargé de cours \\ ${ }^{3}$ Ministère de la Santé Publique du Cameroun, Division de la Recherche Opérationnelle en Santé \\ ${ }^{4}$ International Medical Corps, Cameroun \\ azike21@yahoo.fr
}

\begin{abstract}
La malnutrition est un problème majeur de santé publique en Afrique et au Cameroun. L'étude des pratiques alimentaires des mères permettrait d'identifier et optimiser les interventions à éradiquer la malnutrition infantile. Il s'agissait d'une étude analytique de type cas/témoin avec un échantillonnage de type aléatoire simple. Les sources de données étaient la revue documentaire pour l'obtention de la prévalence de la malnutrition chez les enfants référés au centre nutritionnel ambulatoire, un recrutement consécutif des cas jusqu'à obtention du nombre de cas requis et une recherche communautaire des témoins de ces cas. Au total 386 enfants et leurs mères ont été inclus dans l'étude. La prévalence de la malnutrition infantile référée était de 47,89\% (IC à 95\% [45,58; 50,2]). La classe d'âge la plus touchée était celle des enfants de 6 à 29 mois. Les pratiques alimentaires inappropriées augmentaient significativement le risque de malnutrition chez les enfants avec un $O R=3,44$ [2,05; 5,76] $(P<0,05)$. Après ajustement par les facteurs de confusion nous avons obtenu un OR ajusté $=3,19[2,08 ; 6,35](P=0,0007)$. Les pratiques alimentaires inappropriées les plus retrouvées étaient : les tabous alimentaires, les bouillies non enrichies, l'introduction précoce ou tardive des aliments de compléments. La prévalence de la malnutrition reste très élevée dans le district de santé de Pitoa. Les mauvaises pratiques alimentaires augmentent significativement le risque de malnutrition. Les interventions efficientes seraient l'éducation nutritionnelle et la vulgarisation des aliments locaux riches.
\end{abstract}

Keywords: pratiques alimentaires ; malnutrition ; enfants $<5$ ans ; district de santé dePitoa

\section{Introduction}

La dénutrition ou malnutrition par défaut est l'une des grandes pathologies les plus fréquemment rencontrées dans les pays en développement (PED) identifiées par l'OMS en 1985 (D Lemonnieret, 1986). Elle est le facteur majeur contribuant à augmenter le risque de morbidité et de mortalité infantile. La malnutrition est un facteur complexe causée par plusieurs facteurs : comportementaux, immunitaires et environnementaux. L'alimentation est un facteur clé dans la croissance et le maintien de l'organisme et plus crucial dans la bonne croissance des enfants d'où la tendance mondiale actuelle est basée sur l'alimentation des milles premiers jours de l'enfant. Une bonne alimentation contribuera à éviter la dénutrition, mais une alimentation inappropriée favorisera une installation de la malnutrition pouvant devenir irréversible après le deuxième anniversaire de l'enfant (Unicef New York, 2012).

Les pratiques alimentaires inappropriées contribuent à un apport calorifique et énergétique faible dont à elle seules ou associé à d'autres facteurs peuvent accélérer le processus conduisant à la malnutrition (M. Blössneret, 2005). A celles-ci peuvent se greffer les facteurs socioculturels et socio-économiques qui sont des excellents facteurs sous-jacents de la malnutrition (Pemunta, 2015) ainsi que les facteurs ethniques et religieu x qui n'en demeurent 
pas exclus (Noughani, 2014). D'autres facteurs tels que le niveau de scolarisation et la profession des parents (Kaori, et al, 2007), (Eme, 2014), le nombre d'enfants en charge dans un ménage (Médecin, 2014), la pauvreté et les conditions climatiques en sont des facteurs parallèles susceptibles de conduire également à la dénutrition infantile.

En Afrique subsaharienne, la malnutrition demeure une pathologie grandissante conséquence des conflits politiques. Le Cameroun n'est pas épargné surtout dans sa partie septentrionale où les conditions de vie sont extrêmement difficiles (inondation, sécheresse, etc.) et une situation d'insécurité critique due aux exactions de la secte islamique Boko haram (Unicef et Minsante, 2014). Selon les enquêtes réalisées au Cameroun, seulement $40 \%$ des enfants sont mis au sein dans l'heure qui suit la naissance et $20 \%$ seulement des enfants ont été allaités exclusivement au sein jusqu'à l'âge de 6 mois (Institut National de la Statistique, 2012). La région du nord est la deuxième région ayant un taux élevé de malnutrition selon l'enquête SMART réalisée en 2014 (Unicef et Minsante, 2014). Ainsi dans l'optique de documenter, de fournir aux chercheurs, aux décideurs politiques et sanitaires des connaissances sur les contours de la malnutrition dans le nord Cameroun, nous nous sommes entrepris à mener une étude sur l'association entre la malnutrition et les pratiques alimentaires octroyées aux enfants de 0 à 59 mois dans le district de santé (DS) de Pitoa qui compte dix aires de santé. L'objectif était de déterminer l'association entre les pratiques alimentaires des mères et la malnutrition chez les enfants de 0 à 59 moins dans ce district de santé.

\subsection{Schéma d'étude}

\section{Materiels Et Methode}

Nous avons conduit une étude analytique de type cas-témoins dans la période de juin 2015 à février 2016 afin de déterminer l'association entre les pratiques alimentaires des mères des enfants âgés de 0 à 59 mois et la survenue de la malnutrition chez ces enfants dans le district de santé de Pitoa. A cette étude était nichée une étude transversale pour décrire les pratiques alimentaires de ces mères. Les enfants malnutris âgés de 0 à 59 mois étaient aléatoirement sélectionnés et recrutés dans tous les CNAS/CNAM du district de santé et les enfants témoins étaient recrutés dans leurs communautés respectives par les relais communautaires. Les paramètres nutritionnels étaient mesurés chez ces enfants et leurs mères respectives étaient directement soumises à un questionnaire.

\subsection{Site d'étude}

Le district de Pitoa est un district semi-urbain de la région du Nord Cameroun. Il est traversé par la nationale $\mathrm{n}^{\circ} 1$ qui mène à Maroua chef-lieu de la région de l'Extrême Nord. Le district de santé de Pitoa en particulier et la région du Nord en général appartiennent à la zone soudano-sahélienne qui est caractérisée par un climat tropical chaud et sec, aux précipitations de plus en plus limitées lorsqu'on se rapproche du lac Tchad[10]. La saison de pluvieuse dans cette localité durée seulement trois mois sur douze. Les principales activités de cette population sont : l'agriculture, l'élevage, la pêche et le commerce. Les types aliments les plus consommés étaient céréales (sorgho, maïs, mil), racines et tubercules (patates, ignames), les légumineuses (haricots, arachides, pois de terre), produits laitiers et les produits carnés (très faibles consommation). En 2015, le DS de Pitoa comptait au total 169640 habitants, dont 28670 enfants âgés de 0 à 59 mois. La malnutrition touchait toutes les dix aires de santé et un programme de prise en charge de la malnutrition se fait dans toutes les formations sanitaires 
des aires (publique et privée) à l'aide des intrants fournis par le PAM et l'UNICEF au Cameroun.

\subsection{Echantillonnage}

L'échantillonnage était fait de manière aléatoire stratifiée à deux degrés. Les aires de santé avaient été choisies de manière exhaustive et proportionnellement à leur population de malnutris dans le DS. Au premier degré les CNAS/CNAM étaient sélectionnés de façon randomisée pour le recrutement des cas. Au deuxième degré, les quartiers/villages correspondant aux quartiers/villages de provenance des cas étaient sélectionnés pour le recrutement des témoins. Les mères ou les nourrices de tous les cas et les témoins recrutés étaient ainsi recrutées pour l'évaluation des pratiques alimentaires de leurs enfants.

\subsection{Taille d'échantillon}

La taille d'échantillon avait été estimée à partir de la table 7a de LEMESHOW et LWANGA (1991) de Sample size determination in healthstudies (K. Lwanga, 1991). Pour une probabilité anticipée de «l'exposition » chez les personnes «non malades » de $30 \%$, un rapport de côte anticipé de 2, un niveau de signification de 5\%, une puissance du test de $90 \%$ et une hypothèse alternative que le rapport de côteest différent de 1 , Nous avons obtenu une taille d'échantillon minimale de 176 enfants pour chaque groupe. Nous avons majoré cette taille à $10 \%$ afin de minimiser les erreurs liés aux possibles biais et aux refus de participation. Ainsi, la taille d'échantillon (n) nécessaire pour l'étude était de 388 enfants, soient 194 enfants par groupe.

\subsection{Critères de sélections}

Il s'agissait d'une étude analytique de type cas témoin à laquelle était nichée une étude descriptive de type transversale. Cette étude avait pour objectif principal de déterminer l'association entre les pratiques alimentaires des mères des enfants de 0 à 59 mois et la malnutrition infantile qui sévit dans le DS de Pitoa. Etait considéré comme Cas (enfant malnutri) tout enfant ayant un périmètre brachial dans l'intervalle [115-125] $\mathrm{mm}$ (soit $<115$ $\mathrm{mm}$ (malnutri sévère) ou soit $\geq 115 \mathrm{~mm}$ et $<125 \mathrm{~mm}$ (malnutri modéré)), avec présence ou absence des œdèmes bilatéraux, résidant dans la communauté depuis au moins 2 mois. Comme Témoin (enfant non malnutri) tout enfant de 0 à 59 mois vivant dans la communauté depuis au moins 2 mois, strictement semblable au cas et ayant un périmètre brachial $\geq 125 \mathrm{~mm}$. Etaient considérés comme Exposés les enfants bénéficiant d'une pratique alimentaire inappropriée et comme Non-exposés ceux bénéficiant d'une pratique alimentaire appropriée. Etaient exclusde l'étude, les enfants ayant une pathologie héréditaire telle que la drépanocytose. Une pratique alimentaire est considérée comme appropriée lorsque l'enfant est nourri exclusivement au lait maternel durant les cinq premiers mois, à partir du 6 mois l'introduction des aliments de complément avec diversification alimentaire (Unicef New York, 2012), (OMS, 2009).

\subsection{Collecte des données}

Les données ont été collectées auprès des enfants de 0 à 59 mois et chez leurs mères ou nourrices respectives. Tous les enfants avaient la même probabilité d'être sélectionné. Les enfants sélectionnés étaient introduits dans deux groupes à savoir le groupe des cas et celui des témoins au respect des critères de sélection. 
Les données avaient été collectées à l'aide d'un questionnaire structuré standardisé et pré-testé dans le quartier "Yelwa » à Garoua. Les indicateurs pour évaluer la malnutrition chez l'enfant et les pratiques d'alimentation du nourrisson et du jeune enfant étaient ceux de l'OMS (OMS, 2009) et de l'UNICEF Guide de Programme : Alimentation du Nourrisson et du Jeune Enfant (Unicef New York, 2012).

Vingt-deux (22) relais communautaires servant dans la détection des enfants malnutris dans la communauté avaient été recrutés et recycléspour la détection des témoins au niveau communautaire. Les cas étaient recrutés par l'investigateur principal et vingt-huit (28) personnels de santé (point focal nutrition) des Centres Nutritionnels Ambulatoires pour les malnutris modérés (CNAM) et malnutris sévères (CNAS).Les jours de consultation/distribution étaient fonction du jour du marché de chaque aire de santé (la majorité des centres avait en commun les lundis, mercredis et les jeudis). En fonction du nombre de cas recruté, les témoins étaient respectivement recrutés selon les critères de sélection.

Les données recueillies portaient essentiellement sur les caractéristiques sociodémographiques, les pratiques alimentaires et le statut nutritionnel de leurs enfants. Les matériels utilisés pour le recrutement des participants étaient : « une toise de Shorr » à ruban gradué en centimètre pour la mesure de la taille (une précision de $0,1 \mathrm{~cm}$ ), une balance électronique «SECA » à capacité maximale de $150 \mathrm{~kg}$ pour la mesure du poids (une précision de $100 \mathrm{~g}$ près), le ruban gradué spécial appelé «bande de Shakir » pour la mesure du périmètre brachial. Les données avaient été collectées dans toutes les dix aires de santé du district à savoir : Badjengo, Badjouma Centre, Badjouma Radier, Banaye, Be, Boula Ibbi, Dolla, Holma, Langui Et Pitoa.

Une revue documentaire a été faite sur le masque de données du district de santé de Pitoa de mi 2014 à mi 2015 (une période d'un an). Permettant de ressortir l'évolution de la malnutrition dans le district ainsi que la prévalence.

Les données collectées ont été saisies à l'aide de deux logiciels à savoir EPI Info (version 3.5.4) et ENA 2011 en faisant une double entrée dans chaque logiciel, puis les bases de données étaient comparées et nettoyées. Les principales analyses étaient l'estimation des proportions, des fréquences, des prévalences, des rapports de côtes ainsi que les ajustements avec les facteurs de confusion. Les intervalles de confiance étaient estimés à 95\%.Les tableaux et graphiques ont été conçus à partir d'Excel et de Microsoft Word 2010.

\subsection{Population d'étude}

\section{Resultats}

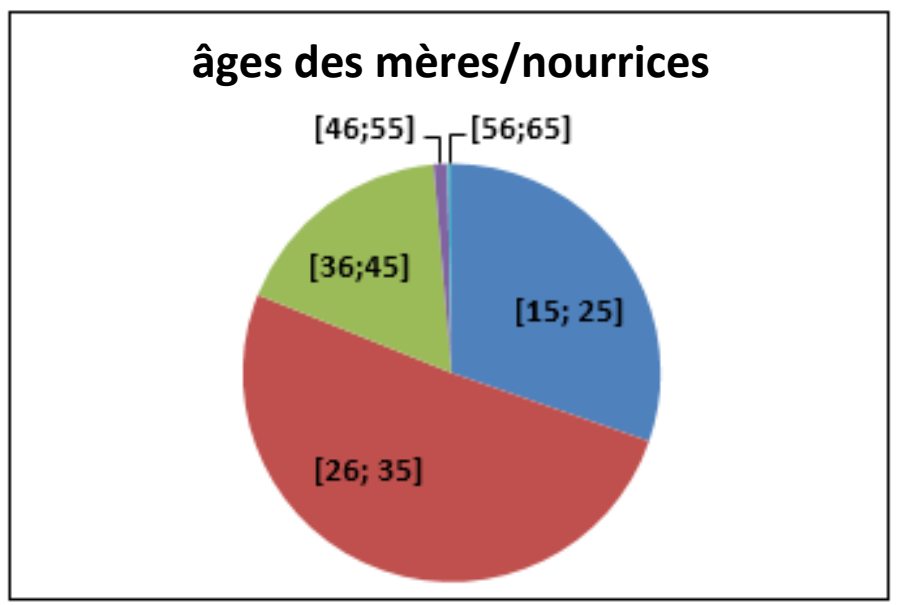

Figure 1. Distribution de l'âge des mères des enfants inclues dans 
Sur les 388 enfants qui devaient participés à cette étude, 2 mères d'enfants avaient refusées de faire inclure leurs enfants ce qui nous donnait un taux de réponse de 99,48\%. La figure 1 présente la distribution par tranche d'âge (année) des mères des enfants ayant participés à l'étude dans le district de santé de Pitoa. La majorité des mères d'enfants étaient encores très jeunes avec un âge compris dans l'intervalle de 15 à 35 ans. Ces femmes exerçaient dans la plus part au moins une activité génératrice de revenue sauf quelques unes qui étaient des ménagères. Les enfants qui avaient été introduits dans l'étude étaient essentiellement âgés de 5 à 59 mois. L'âge moyen des filles cas (malnutris) était de 14,44 mois $[6,67 ; 22,21]$ et celui des filles témoins était de 21,66 [10,94;32,38] mois par contre celui des garçons cas était de 13,32 mois $[7,36 ; 19,28]$ et celui des garçons témoins était de $20,89[9,3 ; 32,48]$ mois. La majorité des enfants étaient dans la tranche de 5 à 25 mois. La répartition en fonction des sexes est identique dans chaque groupe.

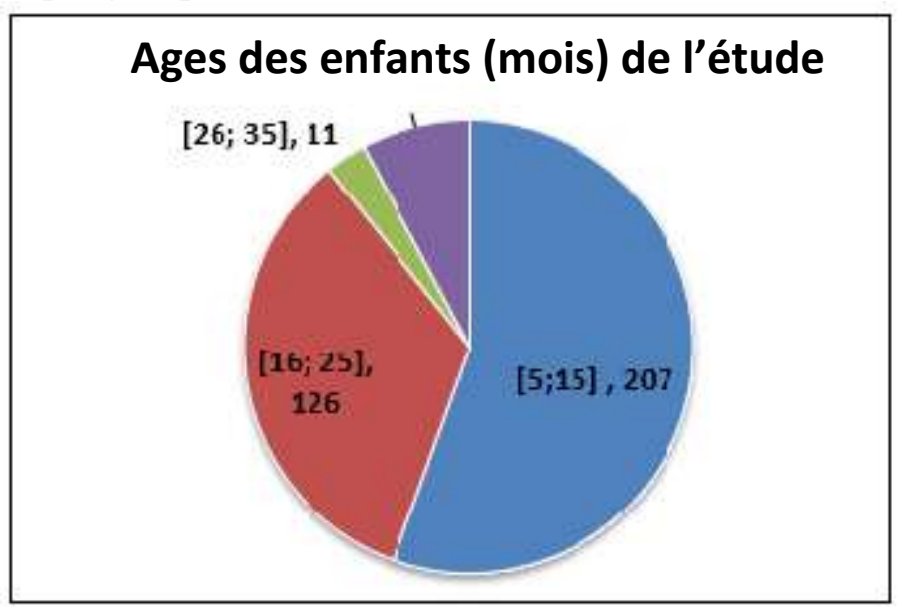

Figure 2. Distribution des âges des enfants inclus dans la population d'étude

La figure 2 quant à elle présente la distribution par tranche d'âge (mois) des enfants ayant participés à l'étude dans le district de santé de Pitoa et le tableau 1 nous présente les caractéristiques sociodémographiques des participants de l'étude.

Tableau 1. Caractéristiques sociodémographiques des mères et des enfants de l'étude dans le district de santé de Pitoa.

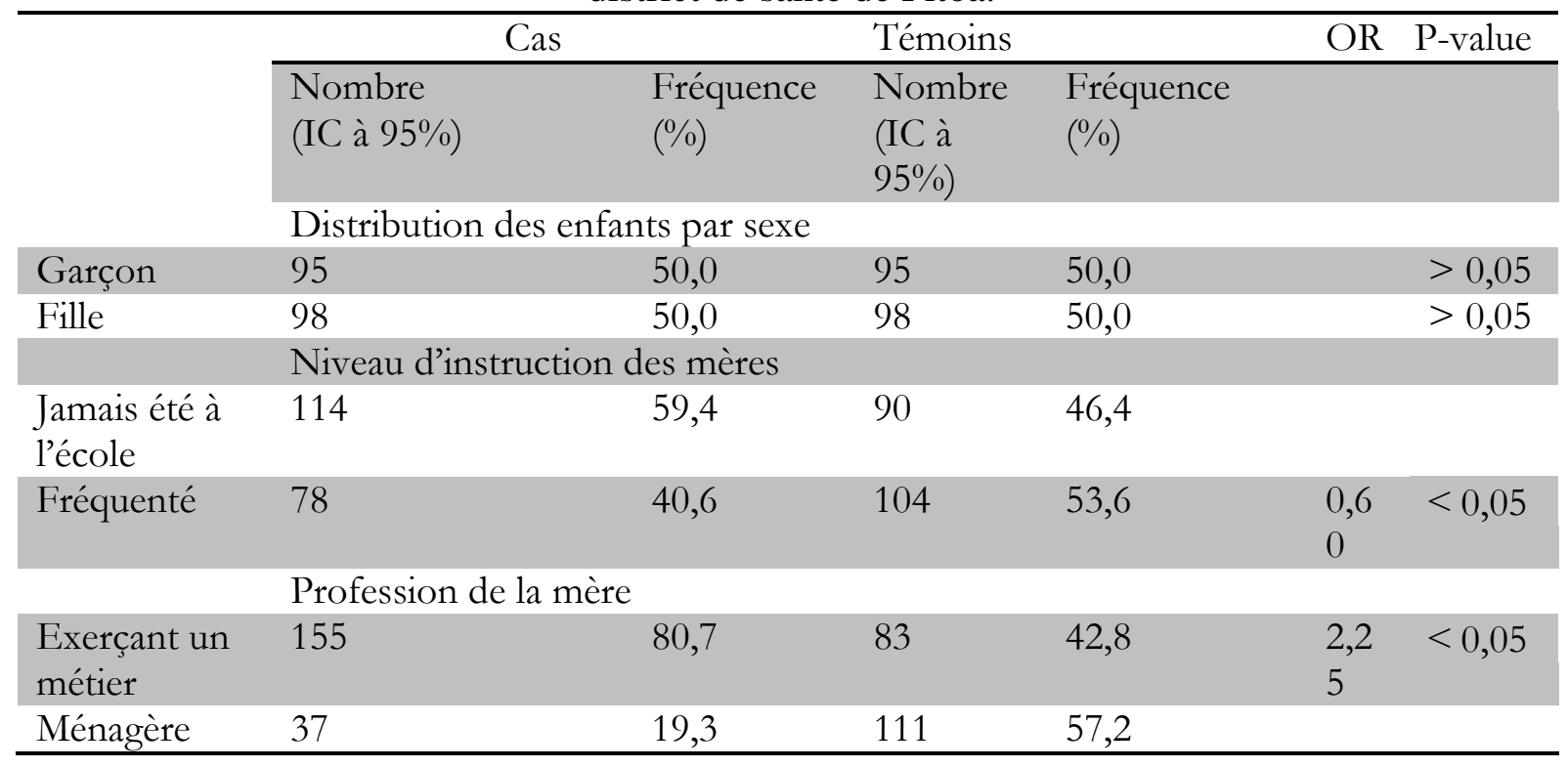




\begin{tabular}{|c|c|c|c|c|}
\hline & Niveau d'instructio & lu père & & \\
\hline $\begin{array}{l}\text { Jamais été à } \\
\text { l'école }\end{array}$ & 88 & 46,8 & 54 & 28,1 \\
\hline Fréquenté & 100 & 53,2 & 138 & 71,9 \\
\hline & Profession du père & & & \\
\hline $\begin{array}{l}\text { Exerçant un } \\
\text { métier }\end{array}$ & 185 & 97,8 & 193 & 100,0 \\
\hline $\begin{array}{l}\text { Non exerçant } \\
\text { un métier }\end{array}$ & 4 & 2,2 & 0 & 0 \\
\hline $\begin{array}{l}\text { Taille } \\
\text { moyenne du } \\
\text { ménage }\end{array}$ & $\begin{array}{l}8,39 \\
{[2,95 ; 13,83]}\end{array}$ & & & $\begin{array}{l}6,91 \\
{[3,34 ; 10,47]}\end{array}$ \\
\hline
\end{tabular}

\subsection{Association entre la malnutrition et les professions, niveau scolaire des parents}

Chez les enfants malnutris, plus de la moitié des mamans n'avaient jamais été à l'école, soit 114/192 (59,37\%) contre 90/194 (46,39\%) chez les témoins. Il existait donc une association significative entre le niveau d'instruction des mères et la survenue de la malnutrition infantile car en effet une mère qui avait au moins été à l'école (minimum le cycle primaire) avait moins de chance d'avoir un enfant malnutri par rapport à celle qui n'avait jamais été à l'école $0 \mathrm{R}=0,60[0,39 ; 0,92](\mathrm{P}=0,0196)$.

Concernant les activités des mères, 155/192 (80,73\%) mères des cas exerçaient au moins une activité génératrice de revenue contre seulement 83/194 (42,78\%) des mères des témoins. Le fait d'exercer les métiers tels que fonctionnaire /salariée, débrouillarde et cultivatrice était associé à la malnutrition chez les enfants. L'association était statistiquement significative pour les cultivatrices. Les enfants des mères cultivatrices avaient six fois plus de risque d'être malnutris $\mathbf{O R}=\mathbf{6 , 9 5}[\mathbf{1 , 8 2} ; \mathbf{2 6 , 5 4}](\mathbf{P = 0 , 0 0 4 6})$ et pour les autres métiers, elle n'était pas statistiquement significative (être débrouillarde : $\mathrm{OR}=2,25(\mathrm{P}=0,42)$; fonctionnaire /salariée : $\mathrm{OR}=3(\mathrm{P}=0,36))$. Il n'existait pas d'association entre le niveau de scolarisation, la profession des pères et la malnutrition. Il était également à constater que la taille des ménages dans cette localité était très élevée soit en moyenne 9 personnes $[2,95 ; \mathbf{1 3 , 8 3}])$ dans les ménages des enfants malnutris contre 7 personnes $[3,34 ; 10,47])$ dans les ménages des témoins. La différence n'était pas statiquement significative (voir tableau 1).

\subsection{Sévérité de la malnutrition chez les enfants référés dans les CNAS et CNAM}

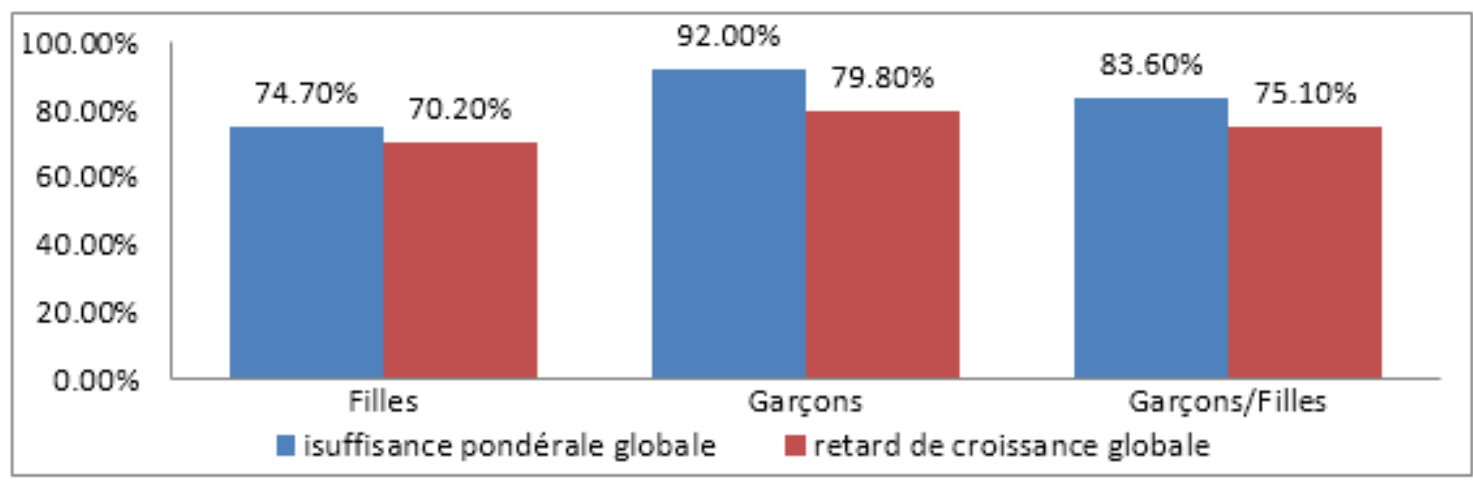

Figure 3. Retard de croissance et insuffisance pondérale chez les 192 enfants malnutris 
Parmi les enfants malnutris référés dans les CNA (CNAS et CNAM) de Juin 2014 à Juin 2015, 47,89 \% étaient malnutris aigües. La Malnutrition Aigüe Modérée (MAM) touchait 27,24\% de ces enfants contre 20,65\% atteints de Malnutrition Aigüe Sévère (MAS).

Selon la figure 3, l'insuffisance pondérale globale (<-2 z-score) touchait 83,6\% des enfants malnutris de l'étude (92,0 \% les garçons et 74,7\% les filles). Dans l'ensemble 50,3\% de ces enfants avaient une insuffisance pondérale sévère (<-3 z-score) dont 60,2\% des garçons et $39,8 \%$ des filles. Le retard de croissance global (<-2 z-score) quant à lui touchait $75,1 \%$ de ces enfants, dont 79,8 \% des garçons et $70,2 \%$ des filles. Sur l'ensemble des enfants atteints de retard de croissance; $43,4 \%$ étaient atteints d'un retard de croissance sévère. Ce retard de croissance s'observait en majorité chez les enfants de 18 à 29 mois, mais il était reparti dans toutes les couches des enfants de 6 à 59 mois. D'après cette figure, on constate que l'insuffisance pondérale et le retard de croissance touchent plus les garçons que les filles (figure $3)$.

\subsection{Les antécédents médicaux (deux mois précédant le début de la malnutrition ou pas) et prévalence de la malnutrition des enfants référés dans les CNAS et CNAM.}

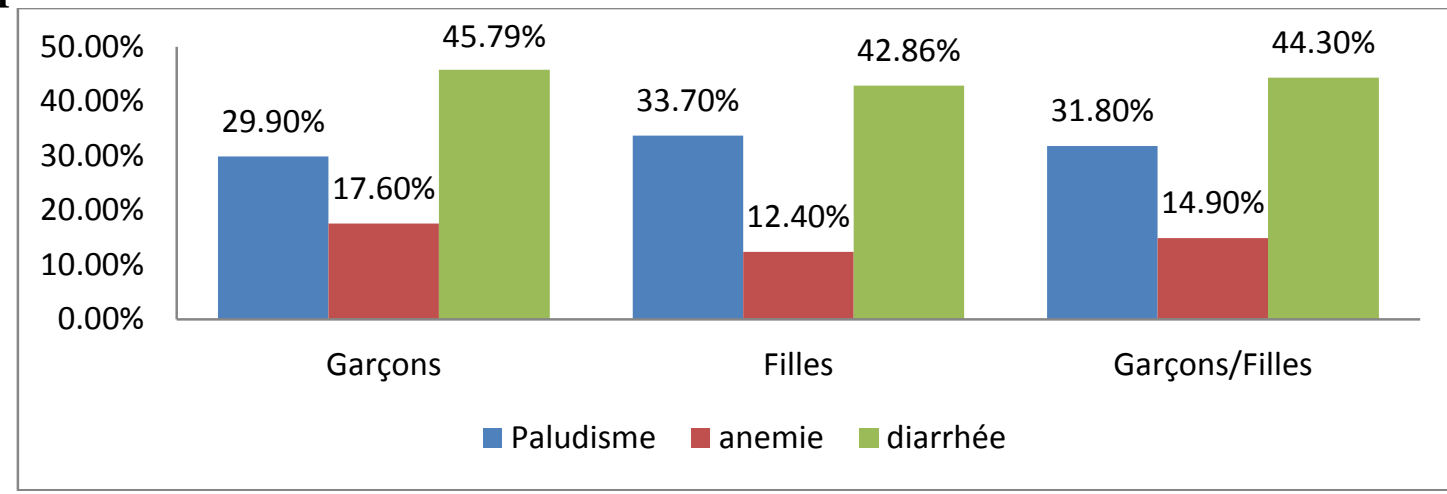

Figure 4. Les antécédents médicaux des enfants introduits deux mois avant l'étude ou avant la détection de la malnutrition.

Nous observions qu'environ trente-deux pourcent $(31,8 \%)$ des enfants avaient été malades du paludisme d'après leurs mères/nourrices avant leur malnutrition (figure 4). Parmi ces enfants qui souffraient de paludisme, la majorité était des filles $(33,7 \%$ étaient des filles contre $29,9 \%$ des garçons). Sur l'ensemble des enfants qui avaient participé à l'étude, 14,9\% présentaient une anémie. Parmi ceux présentant l'anémie, $12,4 \%$ étaient survenu dans la population féminine contre $17,6 \%$ chez les garçons. Dans le groupe des cas, $24,1 \%$ avaient l'anémie contre $5,8 \%$ des témoins qui étaient anémiés. Les garçons avaient 1,5 fois plus de chance de faire une anémie par rapport aux filles, mais cela n'était pas statistiquement significatif $(\mathrm{P}=0,15)$. L'anémie était significativement associée à la malnutrition chez ces enfants dans la population d'étude $(\mathbf{O R}=\mathbf{5 , 1 9} ; \mathbf{2}, \mathbf{5 9} ; \mathbf{1 0 , 3 8}], \mathbf{P}=\mathbf{0 , 0 0 0 1}) .47 / 98(47,96 \%)$ cas parmi les filles avaient eu des épisodes de diarrhée avant la survenue de leur malnutrition contre 37/98 (37,75\%) témoins de sexe féminin qui n'avaient pas développé la malnutrition. 37/95 $(38,95 \%)$ garçons témoins avaient eu des épisodes de diarrhée contre 50/95 $(52,63 \%)$ cas de sexe masculin qui avaient eu les épisodes de diarrhée avant la survenue de la malnutrition chez

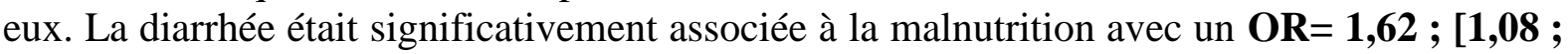
2,44], $(P=0,0185)$. 


\subsection{Pratiques alimentaires et tabous}

Trois cent soixante-dix-huit (378) mères s'étaient prononcées au sujet de l'existence ou non des tabous alimentaires à l'égard des enfants dans leur communauté, 14,3\% (54) de ces mères ont déclaré l'existence de ces tabous alimentaires dans leur communauté. $78,16 \%$ des aliments qui étaient socio culturellement interdits aux enfants étaient d'origine animale (riche en protéine) et l'aliment qui avait été majoritairement interdit dans les communautés était les œufs $(21,68 \%)$.

Quatre-vingt-dix $(47,6 \%)$ cas avaient été allaités au sein pendant 6 mois contre 121 $(62,4 \%)$ témoins qui avaient été allaités au sein durant la même période. 33,3\% des mères pensaient qu'on pouvait donner autre chose que le lait maternel à l'enfant à 6 mois. 39,9\% avaient allaité au sein en dessous des six mois requis et dont $24,6 \%$ d'entre elles avaient fait l'allaitement mixte dès la naissance. $4,3 \%$ par contre avaient allaité exclusivement leur enfant au sein durant la période de 7 mois à 9 mois. Seulement $36,5 \%$ des mères avaient introduit l'alimentation de complément à 6 mois chez l'enfant. 38,4\% avaient introduit précocement l'aliment de complément à leur enfant avant l'âge de 6 mois $(24,7 \%$ l'avaient fait dès la naissance). Et 25,1\% avaient introduit l'aliment de complément tardivement (entre 7 mois et 9 mois). Parmi les aliments de complément introduit aux enfants de moins de 6 mois, il y avait entre autres : l'eau, la bouillie (du sorgho, maïs et du natron), la nourriture, le beignet, les biscuits, le remède traditionnel, le lait de vache.

\subsection{Association entre les pratiques alimentaires des mères et le statut nutritionnel de leurs enfants}

Tableau 2. Tableau de contingence des pratiques alimentaires des mères/nourrices et la survenue de la malnutrition chez les enfants dans le district de santé de Pitoa.

\begin{tabular}{lllll}
\hline & & Cas & Témoin & Total \\
\hline Pratiques & Appropriées & 25 & 66 & 91 \\
alimentaires & Inappropriées & 167 & 128 & 295 \\
& Total & 192 & 194 & 386 \\
\hline
\end{tabular}

Parmi les 386 enfants chez qui nous avons pu évaluer les pratiques alimentaires, 295 $(76,42 \%)$ enfants avaient reçu une pratique alimentaire inappropriée dont 167/295 (56,61\%) étaient des cas et 128/295 (43,38\%) des témoins (tableau 2). Seulement 91/386 (23,58\%) avaient bénéficié d'une pratique alimentaire appropriée soit 25/91 (27,47\%) cas contre 66/91 $(72,52 \%)$ témoins. Les pratiques alimentaires inappropriées des mères étaient significativement liées à la malnutrition chez les enfants de 6 à 59 mois avec un $\mathbf{O R = 3 , 4 4 ; ~ I C ~}$ $[2,06 ; \mathbf{5 , 7 6}](\mathbf{P}<\mathbf{0 , 0 5})$. Après ajustement des pratiques alimentaires avec les facteurs tels que le paludisme, les maladies diarrhéiques, le fait d'avoir déjà reçu un conseil sur les pratiques alimentaires appropriées et les helminthiases; les pratiques alimentaires inappropriées des mères restaient significativement liées à la malnutrition chez les enfants avec OR ajusté= $3,19[2,08 ; 6,35](P=0,0007)$. 


\section{Discussion}

Cette étude menée dans la région du nord Cameroun précisément dans le district de santé de Pitoa a mis en exergue la relation pouvant exister entre les pratiques alimentaires des mères rurales à majorité et la malnutrition qui survient chez leurs enfants.

La prévalence des enfants référés de la malnutrition dans le district de santé de Pitoa était de 47,89\%. Ce résultat avait été obtenu à partir d'une revue documentaire du masque de prise en charge de la malnutrition du district qui était renseigné au premier échelon par les CNA des aires de santé. Cette prévalence bien qu'étant élevée est éloignée de la réalité qui est de 9\% selon l'enquête SMART 2014 pour la région, car la détection des cas se fait dans la communauté par les relais communautaires et qui les orientent vers les CNA dont certaines mères choisissent de venir ou pas. Or le masque de données ne prend en compte que les cas enregistrés dans les CNA. Toutefois cette prévalence n'était pas très différente de la prévalence de 44,7\% obtenue pour la région du Nord publiée par le Journal du Cameroun du 03/03/2014.

Le manque d'instruction et le fait d'exercer un métier chez les mères d'enfant favorisaient la survenue de la malnutrition infantile chez leurs enfants compte tenu du fait qu'une absence d'éducation de la mère est un très grand risque pour l'enfant (soumission aux pratiques des rites, aux tabous alimentaires et us inappropriés au bon développement de l'enfant). Il avait également été démontré à Ibadan au Nigéria, que les mères des enfants malnutris (cas) étaient en grande partie analphabètes ou ayant juste fait le primaire par rapport à ceux des témoins $(35 \%$ contre $26 \%, \mathrm{OR}=3,6[2,0-6,4])[4,7]$.Le fait d'exercer un métier favoriserait la réduction du temps accordé à la prise en charge et au suivi de l'enfant bien que cela favorise l'autonomisation financière. L'enfant est pris en charge par d'autres personnes moins expérimentées, ne maitrisant pas les pratiques alimentaires et les conditions d'hygiène appropriées concernant l'alimentation du nourrisson et du jeune enfant. En inde une corrélation avait été faite entre la profession et la malnutrition; le fait d'être cultivateur et la survenue de la malnutrition aigüe sévère chez l'enfant avait un $\mathrm{OR}=3,36$ avec $\mathrm{p}=0,01$ [6]. Il ne s'agit pas seulement de connaitre les pratiques alimentaires de bases pour la nutrition de l'enfant mais aussi de consacrer du temps à cet effet.

Les pratiques alimentaires inappropriées des mères du district de santé de Pitoa étaient significativement liées à la malnutrition chez les enfants de 6 à 59 mois avec un ORa=3,19 $(\mathrm{P}<0,05)$. Cela veut dire qu'une mère qui pratiquait une pratique alimentaire inappropriée avait trois fois plus de chance que son enfant fasse la malnutrition par rapport à une mère dont l'enfant avait bénéficié d'une pratique alimentaire appropriée. Ce résultat avait été contrôlé par les facteurs de confusion tels que le paludisme, l'assainissement de l'eau, les helminthiases et le fait d'avoir déjà reçu les conseils sur les pratiques alimentaires. Cela veut donc dire que la prévalence de la malnutrition de dépend pas de la prévalence des maladies endémiques telles le paludisme, etc. Un enfant qui bénéficie des pratiques alimentaires appropriées est à l'abri des problèmes nutritionnels même en présence de plusieurs maladies tropicales. Ce résultat allait dans le même sens que les résultats de Tada et al., dans une étude réalisée à Klong Toeyslum (Bangkok) dont les bonnes pratiques alimentaires étaient significativement associées à la malnutrition avec un OR ajusté=0,7123 (95\% CI: 0,5390-0,9414) (Tada Y, 2002).

Bien qu'ayant respecté un chronogramme bien élaboré et une assistance technique diversifiée, cette étude comme la plupart des études cas-témoins a fait face à plusieurs limites méthodologiques telles que les biais de sélection et les biais d'information. Pour les biais de sélection, la sélection des témoins n'était pas forcément dans l'entourage immédiat du patient 
cas correspondant. Néanmoins pour limiter ce biais, tous les témoins étaient recrutés dans le même village/quartier, dans le voisinage le plus proche possible que le cas correspondant.

Dans la revue documentaire, il n'y avait pas des données sur l'âge, le sexe, la taille et le périmètre brachial de chaque enfant permettant d'estimer les retards de croissance, l'insuffisance pondérale ainsi que la distribution de la malnutrition chez ces enfants pendant cette année. Les biais d'informations avaient été contrôlés lors de la collecte des données par le fait que les enquêteurs parlaient couramment français et le foulbé (langue locale) pour minimiser les biais dus à la traduction. Et les données collectées par les enquêteurs étaient vérifiées par l'investigateur principal et chaque enquêteur pour vérification de la complétude et exactitude afin de minimiser les biais de lecture.

\section{Conclusion}

La malnutrition infantile dans ce district a pour principale cause les pratiques alimentaires inappropriées. En effet, un enfant qui ne bénéficie pas de pratiques alimentaires appropriées a trois plus de chance d'être malnutris. Le paludisme, les diarrhées à répétition et les anémies ne sont pas à négliger non plus dans les facteurs d'aggravation et d'installation de la malnutrition.

Nous recommandons, une intensification de l'éducation nutritionnelle des mamans dans cette région. Une promotion de l'éducation scolaire des femmes de cette région et l'introduction de l'éducation nutritionnelle dans les programmes scolaires dès le primaire. Une recherche approfondie des constituants nutritionnels des aliments locaux pour une formation pratique. Un appui externe pour la prise en charge améliorée des cas prévalent. La promotion de l'éducation nutritionnelle dans les tontines, les gics et dans les centres de la promotion de la femme. Une intégration des pères dans le processus de prise en charge des enfants malnutris afin que ce ne soit uniquement le problème de la mère. De préconiser également les causeries éducatives sur le thème de la nutrition dans les quartiers et villages serait d'un très grand apport pour éradiquer ce fléau du Cameroun.

\section{References}

D Lemonnieret Y lngenbleek. (1986). Les Malnutritions Dans Les Pays Du Tiers-Monde : Anemie Ft Malnutrition Proteino-Energetique Moderee Chez L'enfant De La Province Du Nord-Cameroun. Edition Inserm, 121-132.

Eme Owoaje., Oluwadolapo Onifadeet Adeyimika Desmennu. (2014). Family and Socio Economic Risk Factors for Undernutrition Among Children Aged 6 to 23 Months in Ibadan, Nigeria. The Pan AfricanMedical Journal, 161.

Institut National de la Statistique. (2012). EDS-MIC 2011, chapitre 10 : Etat nutritionnel, Allaitement et pratiques alimentaires, Page 171.

Kaori Saito., Joshua R. Korzenik., James F. Jekelet Sara Bhattacharji. (1997). a Case-Control Study of Maternal Knowledge Malnutrition and Health-Care-Seeking Attitudes in Rural South India. Yale Journal Of Biology And Medecine, 149-16.

M. Blössneret Mercedes de Onis. (2005). Malnutrition: Quantifying the Health Impact at National and Local Levels. Geneva. WHO Environmental Burden of Disease Series, No. 12.

Médecin du Monde. (2014). L'alimentation Des Personnes En Situation De Grande Precarite En France, Publié en Juin. 
Noughani F., Bagheri M. etRamim T., (2014). Nutritional habits of mothers and children in the age group 0-4 years in Iran. Ecol Food Nutrion, 410-8.

OMS. (2009). Indicateurs pour évaluer les pratiques d'alimentation du nourrisson et du jeune enfant, Première partie Définitions, Conclusions d'une réunion de consensus du 6 au 8 novembre 2007, à Washington, D.C., États-Unis d'Amérique.

Pemunta N.V. et Fubah M.A., (2015). Socio-cultural determinants of infant malnutrition in Cameroon. JbiosocSci, 423-48.

S. K. Lwanga., \& S. Lemeshow. (1991). Sample Size Determination In Health Studies: A Practical Manual, World Health Organization Geneva, 10-11.

Tada Y., Keiwkarnka B., Pancharuniti N. et Chamroonsawasdi K., (2002). Nutritional status of the preschool children of the KlongToey slum, Bangkok.Southeast Asian J Trop Med Public Health. Sep; 33(3):628-37.

Unicef Et Minsante. (2014) .Situation nutritionnelle au Cameroun (EN, NO, AD et Est), Enquête Smart, 11.

Unicef New York. (2012). Guide de Programme : Alimentation du Nourrisson et du Jeune Enfant, page 3-4, Juin 2012 http://www.unicef.org/nutrition/ 